\title{
A METAMORFOSE DO SISTEMA DE RECURSOS DE LICENCIANDOS EM MATEMÁTICA NA FORMAÇÃO INICIAL
}

\author{
THE METAMORPHOSIS OF PROSPECTIVE MATH TEACHERS' \\ RESOURCE SYSTEM IN THE INITIAL TRAINING \\ Cibelle Assis ${ }^{1}$
}

Universidade Federal da Paraíba - UFPB

Verônica Gitirana ${ }^{2}$

Universidade Federal de Pernambuco - UFPE

Luc Trouche ${ }^{3}$

École Normale Supérieure de Lyon

\begin{abstract}
Resumo
Neste artigo apresentamos parte de um estudo desenvolvido durante o Estágio de PósDoutorado da autora sob supervisão dos coautores entre os anos de 2017 e 2018. O objetivo deste estudo foi identificar aspectos do desenvolvimento profissional de licenciandos em matemática através da análise do seu trabalho com recursos. Como referencial teórico e metodológico nós nos apoiamos na Abordagem Documental do Didático. Realizamos uma Investigação Reflexiva e um Estudo de Caso com uma licencianda cujos dados obtidos foram analisados numa perspectiva qualitativa. A pesquisa compreendeu quatro fases: estudo e representação da Trajetória Documental da licencianda; coleta e análise de tarefas matemáticas em situações de estudo e de ensino; entrevistas com a licencianda; e a análise da atividade da licencianda durante a implementação de uma aula em uma escola. Podemos afirmar que a constituição do sistema de recursos para ensinar da licencianda pode ser compreendido através de um processo que denominamos de metamorfose. Em síntese, o caso estudado contribuiu para identificar recursos de transição e profissionais como aspectos importantes da formação além de uma percepção diferenciada para os recursos e seus usos, quer sejam pelos licenciandos e seus professores dos cursos de formação ou professores da escola. A análise da Trajetória Documental permitiu um panorama geral da formação do licenciando e da própria Licenciatura, assim como a necessidade do acompanhamento, pela equipe de professores envolvidos na formação, do processo de metamorfose dos licenciandos e suas especificidades.
\end{abstract}

Palavras-chave: Recurso; Metamorfose; Abordagem Documental do Didático; Formação de professores; Matemática.

\section{Abstract}

This article was part of a study developed during the post-doctoral course on the supervision of coauthors between the years 2017 and 2018. The aim of this study was to identify aspects of the professional development of prospective teachers through the

\footnotetext{
1 cibelle@dce.ufpb.br

2 veronica.gitirana@gmail.com

3 luc.trouche@ens-lyon.fr
} 
analysis of their work with resources during a math education initial training course. As a theoretical and methodological framework, we based on the Documentational Approach to Didactics. We conducted a Reflective Investigation and a Case Study with a prospective teacher, from a qualitative perspective. The research comprised four phases: study and representation of the Documentational Trajectory; collection and analysis of mathematical tasks in study and teaching situations; interviews; analysis of the prospective's activity during the implementation of a class in a school. We assume which the constitution of the system of resources can be understood through a process that we call metamorphosis. In summary, the case studied contributed to identify transactional and professionals resources as the important issues of the training of the prospective teacher and to a differentiated perception of resources and their uses by prospective teachers, teachers from university or from school. In addition, the Documentational Trajectory brought an overview of the student's training and of the course itself, as well as the need to follow the process of metamorphosis, by the team of teachers engaged in it, of the students and their specificities.

Keywords: Resource; Metamorphosis; Documentational Approach to Didactics; Teacher training; Mathematics.

\section{Contexto e problemática da pesquisa}

Acreditamos que a formação inicial é um momento importante da trajetória do professor. É durante essa experiência, por exemplo, que são desenvolvidos conhecimentos formais necessários à profissão. De acordo com Ponte e Brunheira (2001), essa formação precisa levar em conta todas as dimensões do conhecimento profissional e deve considerar a maneira como os estudantes podem desenvolvê-las. Como elementos importantes deste processo, consideramos a constituição, a estruturação e a evolução do sistema de recursos desses futuros professores.

Sobre os recursos de ensino, Adler (2000) os conceitua como tudo o que nutre ou origina a atividade docente. Por exemplo, os professores usam recursos de diferentes naturezas para objetivos diferentes, sejam para apoiar a aprendizagem de seus alunos ou para dar suporte ao ensino. Assim, eles mobilizam vários livros didáticos e apoiam-se em referenciais curriculares; elaboram listas de exercícios; integram jogos matemáticos e calculadoras em suas aulas; utilizam-se do quadro branco e do projetor de imagens; e fazem uso do computador e de softwares educacionais, além de materiais disponíveis na internet, como vídeos, aulas ou atividades compartilhadas por outros professores.

No contexto da formação inicial, o trabalho com recursos alimenta uma problemática que consiste em entender como os licenciandos constituem seus primeiros sistemas de recursos. Temos observado que esses estudantes passam a "adotar" alguns 
Cibelle Assis, Verônica Gitirana, Luc Trouche

A metamorfose do sistema de recursos de licenciandos em matemática na formação inicial

recursos particulares e que tais recursos promovem novas formas de aprender matemática e de desenvolver conhecimentos profissionais. Este foi o caso do software Geogebra para alguns dos estudantes da Licenciatura que acompanhamos: um recurso desconhecido na época em que cursaram a educação básica e que passou a integrar não apenas o repertório de recursos para aprender matemática na Licenciatura mas, também, para conceber propostas de ensino que posteriormente foram desenvolvidas nas escolas (através dos estágios supervisionados, dos projetos de ensino e de trabalhos de conclusão de curso).

A pesquisa que apresentamos neste artigo surgiu da nossa experiência enquanto professores da Licenciatura em Matemática e da necessidade de compreensão dos processos formativos dos estudantes nesta etapa profissional. Temos como objetivo discutir aspectos do desenvolvimento profissional de licenciandos em matemática através da análise do trabalho que realizam com recursos, mais especificamente, quais fatores influenciam a constituição do seu sistema de recursos.

Para tal questão apresentamos duas hipóteses: 1) o sistema de recursos para ensinar - SREns se constitui a partir do sistema de recursos para estudar - SREst que os estudantes constroem desde a escola passando pelo período de formação na universidade; 2) esse processo de mudança do SREst para SREns se alimenta tanto da mobilização de conhecimentos profissionais já adquiridos como também de novos conhecimentos mobilizados no processo. Nesses termos, apresentamos a ideia de metamorfose do sistema de recursos para conceituar o processo de mudança do SREst para SREns.

Após esta breve contextualização e apresentação da nossa problemática, nas seções seguintes faremos uma introdução da Abordagem Documental do Didático ADD discutida por Gueudet e Trouche $(2008,2009)$ principalmente das noções de recurso, documento e gênese documental, e como o termo metamorfose foi construído a partir desta perspetiva. Posteriormente, faremos nossas considerações sobre as escolhas metodológicas assim como sobre o sujeito Cecília (nome fictício da licencianda) do estudo de caso desenvolvido. Nas demais seções discutiremos a metamorfose de Cecília e como tais aspectos relativos ao desenvolvimento profissional dessa licencianda podem iluminar uma perspectiva de análise, através dos recursos, para a formação inicial de outros estudantes assim como para os cursos de formação. 
Cibelle Assis, Verônica Gitirana, Luc Trouche

A metamorfose do sistema de recursos de licenciandos em matemática na formação inicial

\section{A Abordagem Documental do Didático}

O trabalho do professor é dinâmico e se refaz à medida que ele reflete sobre a sua própria prática. Nesse processo, as tarefas matemáticas já aplicadas podem ser reformuladas conforme as experiências vivenciadas com seus estudantes em situações de sala de aula; um plano de aula pode ser modificado a partir da interação com outros professores; os recursos antigos podem ganhar outros significados e novas formas de utilização ao longo do tempo.

Para Gueudet e Trouche (2009, p. 199), as ações do professor neste movimento de ressignificação e de revisita de seus recursos e de suas produções, estão no "centro" da atividade e do desenvolvimento profissional do professor de Matemática. Para esse conjunto de ações em que os professores transformam de maneira significativa seus recursos e, como produto, constituem seus documentos, denomina-se Trabalho Documental do professor e, nesta perspectiva, sua análise é denominada de Abordagem Documental do Didático - ADD (GUEUDET; TROUCHE, 2009).

A Abordagem Documental do Didático se alimenta de outros quadros teóricos, alguns da Educação Matemática como a Teoria das Situações Didáticas de Brousseau (1998), a Teoria Antropológica do Didático de Chevallard (2002) e a Teoria dos Campos Conceituais de Vergnaud (1996), e de outros quadros, como a Teoria Ergonômica de Rabardel (1995) que discute a inclusão de instrumentos no campo da Didática.

A Abordagem Documental do Didático conceitua e diferencia recurso de documento evidenciando a dinâmica da construção do documento como elemento da atividade profissional do professor. Para Gueudet e Trouche (2009), existe uma relação dialética entre recurso e documento. Segundo os autores, esse processo pode ser representado por uma espiral (Figura 1) em torno de um eixo vertical que representa o tempo, na qual a elaboração de um documento (documento 1) provém da utilização de um conjunto de recursos (conjunto de recursos 1). Esse documento, por sua vez, pode constituir um novo conjunto de recursos (conjunto de recursos 2) para a geração de um novo documento (documento 2). 


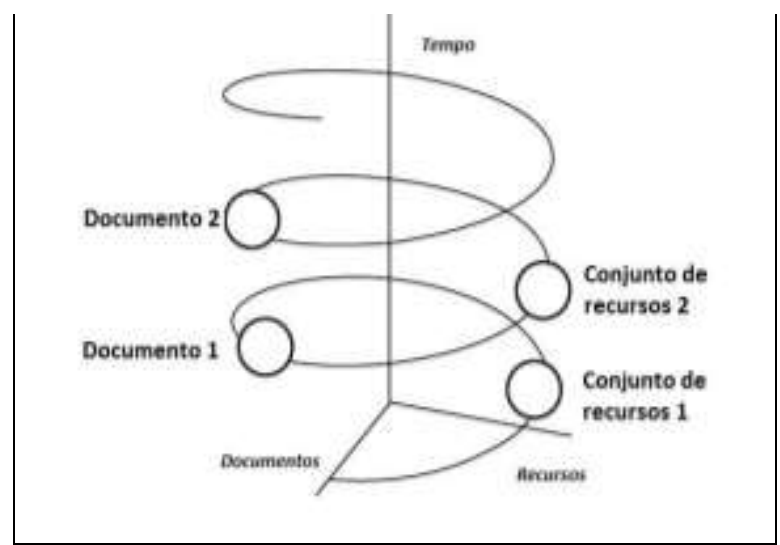

Figura 1 - Relação dialética entre recurso e documento Fonte: Adaptado e traduzido de Gueudet e Trouche (2009, p. 8)

Seguindo Adler (2000), Gueudet e Trouche (2008) propõem uma noção de recursos com algumas restrições: o conhecimento do professor não seria considerado um recurso (e sim o que orienta seu trabalho com o recurso); da mesma forma, os colegas de trabalho do professor não seriam recursos (e sim os conselhos, mensagens e propostas desses colegas), portanto, somente seriam considerados recursos as entidades materiais ou materializáveis.

Para Bellemain e Trouche (2016) um recurso pode ser de diferentes níveis: recursos "conteúdo" (livro didático), recursos "metarrecursos" que facilitam o acesso ao primeiro nível de recursos (sites de busca que levam a vídeos, por exemplo) e finalmente recursos para "trabalhar o conteúdo" (editores de texto, softwares de geometria).

\section{A Gênese Documental}

Essencialmente, a perspectiva documental distingue o que está disponível para a atividade dos professores (os recursos) e o que eles desenvolvem, constroem ou criam para apoiar a sua atividade de ensino (os documentos). Esse processo de transformação é chamado de gênese documental.

A gênese documental comporta duas dimensões. Uma que trata da influência dos recursos (possibilidades, limitações, restrições, condições) na atividade do professor (professor $\rightarrow$ recurso) e, a outra, da apropriação e (re)criação dos recursos pelo professor modificando-os para seu uso (recurso $\rightarrow$ professor). Guin e Trouche (1999) denominaram essas duas dimensões de instrumentação e instrumentalização, 
Cibelle Assis, Verônica Gitirana, Luc Trouche

A metamorfose do sistema de recursos de licenciandos em matemática na formação inicial

respectivamente. A Figura 2, a seguir, ilustra de forma esquemática a gênese documental.

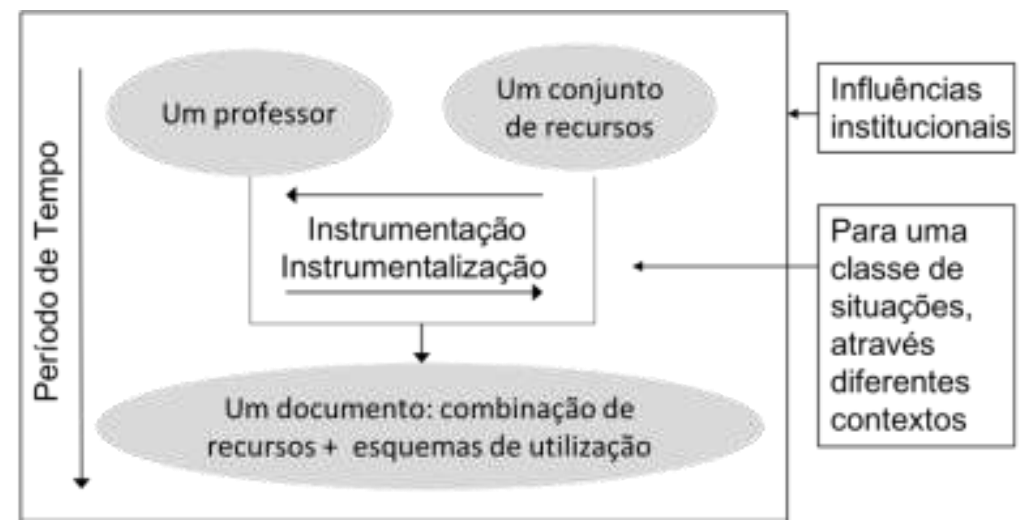

Figura 2 - Representação esquemática da gênese de um documento Fonte: Adaptado e traduzido de Gueudet e Trouche (2009, p. 206)

A partir da figura observamos que um documento reflete de alguma forma os conhecimentos do professor, suas concepções de ensino e de aprendizagem, seus modos de trabalho nos contextos coletivos e institucionais nos quais atua, considerando sua experiência ao longo do tempo. Assim, um documento é um produto dos recursos disponíveis a esse professor associado aos esquemas de utilização desses recursos em diferentes situações de atividade ou classes de situações (por exemplo, planejamentos de aulas, de tarefas ou preparação de uma avaliação sobre conteúdo específico). Gueudet e Trouche (2011) representam o processo de produção de um documento pela estrutura: documento $=$ recurso + esquemas de utilização.

No campo profissional, Goigoux e Vergnaud (2005, p. 7) conceituam "esquema" como "uma forma organizada e estabilizada da atividade docente para uma certa variedade de situações pertencentes à mesma classe". Ou seja, os esquemas podem ser identificados quando são percebidas regularidades na ação do professor referente ao uso do recurso para uma mesma situação de aula em diferentes contextos. São os esquemas que orientam o trabalho sobre os recursos mobilizados para um dado objetivo de ensino (GUEUDET; TROUCHE, 2008, p. 11).

\footnotetext{
4 A noção de esquema foi inicialmente introduzida por Piaget em 1936, com aceitação na Educação Matemática, especialmente na Didática da Matemática (Teoria dos Campos Conceituais) com Gérard Vergnaud (1993). Para descrever mais precisamente a atividade de pensamento de um professor em uma situação de trabalho, Goigoux e Vergnaud (2005) usam uma segunda definição de esquema analisando-a a partir de quatro elementos: o objetivo, as regras (de ação, de tomada de informação e controle), invariantes operatórios (conceito-em-ação e teorema-em-ação) e inferências.
} 
Cibelle Assis, Verônica Gitirana, Luc Trouche

A metamorfose do sistema de recursos de licenciandos em matemática na formação inicial

O termo "utilização" referente ao esquema, segundo Bellemain e Trouche (2016), deve ser entendido em sentido amplo: "trata-se de toda a ação didática do professor, desde a seleção dos recursos até sua adaptação, sua estruturação, sua implementação na sala de aula, sua revisão a posteriori, etc.” (BELLEMAIN; TROUCHE, 2016, p. 11).

A elaboração de um documento (por exemplo, de uma tarefa) ocorre a partir de diferentes esquemas de utilização que são planejados pelo professor para esta nova situação. No entanto, eles se modificam no momento de sua execução, na interação com os alunos e no ambiente escolar. Além disso, a análise da ação passada possivelmente gerará modificações e adequações nessa nova utilização em sala, transformando um documento antigo em um recurso para um novo documento. Esses aspectos podem ser identificados a partir de uma análise conjunta com o professor, resgatando as transformações e usos desse documento ao longo do tempo.

No entanto, existem aspectos não observáveis nos esquemas de utilização do documento chamados de invariantes operatórios, mas que podem ser inferidos a partir dos conhecimentos, das concepções, das crenças do professor sobre o processo de ensino e aprendizagem que estão fortemente presentes na sua prática docente (nos seus documentos e na sua ação em sala de aula). Os invariantes operatórios são a base dos esquemas e orientam essa utilização dos recursos. Segundo Bellemain e Trouche (2016), eles se caracterizam por serem:

[...] relativamente resistentes (eles se formaram ao longo de vários ciclos de uso). Eles também têm alguma plasticidade, e podem evoluir no confronto com as novas circunstâncias de ensino (novos recursos disponíveis, desempenho didático na sala de aula, etc.). (BELLEMAIN; TROUCHE, 2016, p. 11).

Entendemos que a análise isolada de uma produção de um professor, como um plano de aula ou mesmo uma tarefa, não é suficiente para descrever o seu trabalho documental. Essa análise envolve uma complexidade de elementos, alguns não observáveis e outros que estão apenas no registro da memória do professor ou diluídos no tempo. Para Gueudet e Trouche (2009), um documento é um indicativo do desenvolvimento profissional do professor. É neste sentido que a Abordagem Documental analisa o trabalho dos professores por meio das diversas gêneses documentais tecendo os fios desse processo, sendo alguns até mesmo inconscientes ao professor. 
Cibelle Assis, Verônica Gitirana, Luc Trouche

A metamorfose do sistema de recursos de licenciandos em matemática na formação inicial

Para estudar o trabalho de documental de professores, Gueudet e Trouche (2012) propuseram a metodologia de Investigação Reflexiva. Os princípios subjacentes a esta metodologia são: acompanhamento de longo prazo do trabalho do professor, acompanhamento em sala e fora da sala de aula, ampla coleta de recursos materiais e acompanhamento reflexivo.

$\mathrm{O}$ último princípio indica um elemento reflexivo à metodologia, uma vez que o professor participa da coleta de dados, contribuindo para a análise de seu próprio trabalho documental. Trata-se de recuperar com a colaboração do professor "elementos de continuidade de seu trabalho documental em uma diversidade de lugares e momentos.” (BELLEMAIN; TROUCHE, 2016, p. 12).

Mais recentemente, Rocha e Trouche (2017) propuseram o termo "mapeamento" dos recursos do professor integrando uma ideia de exploração progressiva de um novo território em vez da simples ideia de representação. Os autores também diferenciam o mapeamento reflexivo (criado pelo próprio professor) daquele inferido (feito pelo pesquisador) a partir dos dados e informações coletadas.

Como resultado, os autores definiram a Trajetória Documental do professor como a interação entre eventos e recursos, ou a história entre eventos e recursos que pode ser representada de forma esquemática seguindo uma linha do tempo. Nesta perspectiva, um recurso é algo que dá suporte ao trabalho do professor e um evento é definido como algo que aconteceu na vida profissional do professor e que, no momento da elaboração da trajetória, ele lembrou como importante em relação ao seu trabalho de documentação.

Para Rocha e Trouche (2017), traçar a Trajetória Documental de um professor é uma maneira de analisar em que momento e quais recursos foram criados e usados ao longo do tempo, mas principalmente uma maneira de analisar o desenvolvimento profissional a partir dos recursos do professor. Para o desenho e análise de uma trajetória documental, os autores indicam o uso de entrevistas, do diário de bordo preenchido por professores, e também acompanhamento da preparação da aula e observação em sala de aula.

\section{A Metamorfose do sistema de recursos}

Nesta seção apresentamos nossas próprias proposições de acordo com a ADD. Introduzimos as noções de "sistema de recursos para estudar", "sistema de recursos para 
Cibelle Assis, Verônica Gitirana, Luc Trouche

A metamorfose do sistema de recursos de licenciandos em matemática na formação inicial

ensino" e "metamorfose" do sistema de recursos, permitindo conceituar a forma como os futuros professores constituem seus sistemas de recursos, considerando o contexto de um curso de formação inicial de professores.

Com base em Besnier (2016), distinguimos, para os futuros professores, duas principais classes de situações: situações de estudo e situações de ensino. As situações de estudo compreendem experiências relacionadas ao estudo de qualquer assunto durante o curso, por exemplo: de matemática, de um software específico ou como preparar um plano de aula. As situações de ensino consideram experiências na universidade ou na escola, com colegas ou alunos como público. Correspondendo a estas duas classes, para um determinado licenciando, temos um sistema de recursos para estudar (SREst) e um sistema de recursos para ensinar (SREns). O SREst considera tudo que apóia a atividade de estudo do licenciando e, o SREns, as fontes que apóiam o seu ensino de matemática.

Também consideramos que o conjunto de recursos tem uma estrutura própria que permite nomeá-lo como um sistema. Além disso, há uma coerência entre eles que nos levou a caracterizar mudanças do SREst para SREns e denominá-las de metamorfose. Analisamos essas mudanças seguindo três dimensões: o conteúdo, a estrutura e a natureza.

Em relação ao conteúdo, a metamorfose é analisada através de uma perspectiva material. Isto é, significa identificar recursos materiais utilizados durante o desenvolvimento de um conjunto de atividades em longo ou curto prazo de tempo. Para isso, buscamos responder questões como: “que recursos?", “que tipo?”, “quando eles foram usados?", e “usados para ensinar ou para estudar?". Na prática, significa inferir uma primeira versão para o SREst e SREns.

$\mathrm{O}$ estudo sobre estrutura revela novas relações entre os recursos e (re) estabelecimento de seus papéis. Seguindo Besnier (2016), uma vez que os recursos são organizados de acordo com blocos específicos de atividades relacionadas às classes de situações, pode-se inferir os papéis desempenhados pelos recursos. No nosso caso, as classes foram estudar e ensinar (ex. Realizando uma tarefa com Geogebra e Criando uma tarefa com Geogebra, respectivamente) e inferimos mudanças sobre os SREst e SREns. Portanto, além de identificar recursos, essa análise infere os papéis desempenhados: se um papel particular em uma situação particular ou em muitas 
Cibelle Assis, Verônica Gitirana, Luc Trouche

A metamorfose do sistema de recursos de licenciandos em matemática na formação inicial

situações, durante um longo ou curto período de tempo. Nesta dimensão tentamos responder as perguntas: "recursos para quê?" e "quais funções para quais recursos?".

Por fim, o estudo sobre a natureza visa identificar a mudança do status "aluno" para o status "professor" de um futuro professor através da integração com seus recursos, a fim de realizar uma atividade profissional. Significa investigar o que eles fazem com seus recursos e por quê. Esta consideração revela esquemas de utilização dos recursos, conhecimentos profissionais associados e competências desenvolvidas. No entanto, usando o conceito de esquema de acordo com Goigoux e Vergnaud (2005), estudar a natureza da metamorfose para nós é estudar esquemas profissionais relacionados a uma situação de ensino. Consideramos uma situação de ensino como todas as ações didáticas do professor que compreende, por exemplo, selecionar, adaptar e estruturar os recursos, não apenas implementando-os na aula. A análise dessas três dimensões permite compreender o modo como os futuros professores constituem seus sistemas de recursos.

\section{Considerações metodológicas}

Neste artigo apresentamos parte de um estudo desenvolvido durante o Estágio de Pós-Doutorado da autora sob supervisão dos coautores entre os anos de 2017 e 2018. Os resultados são referentes a um estudo de caso (YIN, 2005) desenvolvido com uma estudante da Licenciatura em Matemática da Universidade Federal da Paraíba. Aplicamos a Metodologia da Investigação Reflexiva seguindo Gueudet e Trouche (2012), entre os meses de março e junho de 2016 observando dados e informações sobre o trabalho documental realizado pela licencianda entre os anos de 2011 e 2016.

A estudante nomeada por nós de Cecília, foi escolhida por duas razões principais: (1) no momento da coleta de dados estava em seu último período e, portanto, vivenciou uma variedade de eventos (disciplinas e projetos) ocorridos durante o curso de Licenciatura que lhe permitiram traçar um panorama da sua formação em termos de experiências e recursos; (2) teve o Geogebra como recurso utilizado para estudar e para ensinar matemática, o que nos proporocionou estudar a transição e constituição de um sistema de recursos em momentos diferentes da sua trajetória.

A metodologia da pesquisa foi organizada em quatro fases. Primeiramente, Cecilia foi convidada a construir sua Trajetória Documental (ROCHA; TROUCHE, 2017) seguida de uma entrevista para relacionar eventos e recursos importantes para o 
Cibelle Assis, Verônica Gitirana, Luc Trouche

A metamorfose do sistema de recursos de licenciandos em matemática na formação inicial

seu desenvolvimento profissional mencionados na Trajetória. A entrevista foi realizada em novembro de 2016 e foi gravada em áudio e vídeo. Em seguida, foi solicitado a Cecilia que enviasse aos pesquisadores, por e-mail, algumas tarefas previamente desenvolvidas com o Geogebra, tanto para o estudo como para o ensino de matemática. Esta coleta de dados foi realizada em julho de 2017. O conjunto de tarefas coletadas foi analisado considerando os documentos que ela produziu/utilizou, como planos de aula, relatórios, bem como gravações da implementação de algumas aulas na escola nas quais as tarefas e documentos foram utilizados. Esse conjunto de dados, organizado de acordo com cada tarefa, foi denominado Grupo de Recursos (GR).

Em seguida, passamos para as análises quanti-qualitativas dos dados de maneira que fosse possível identificar e compreender as dimensões conteúdo, estrutura e natureza da metamorfose de Cecília. Para tanto nos apoiamos na ADD para compor as dimensões e analisamos em termos de esquemas, segundo Vergnaud (1993), os elementos da dimensão natureza fornecidos a partir do vídeo da aula implementada.

\section{A Metamorfose do sistema de recursos de Cecília}

A combinação de dados reflexivos de Cecília e as nossas inferências permitiram representar os sistemas de recursos para estudar e ensinar a partir do desenvolvimento com atividades apoiadas pelo Geogebra, e identificar os papéis desempenhados pelos recursos neste momento específico da metamorfose. Iniciamos por uma representação para a Trajetória Documental inferida de Cecília (Figura 3).

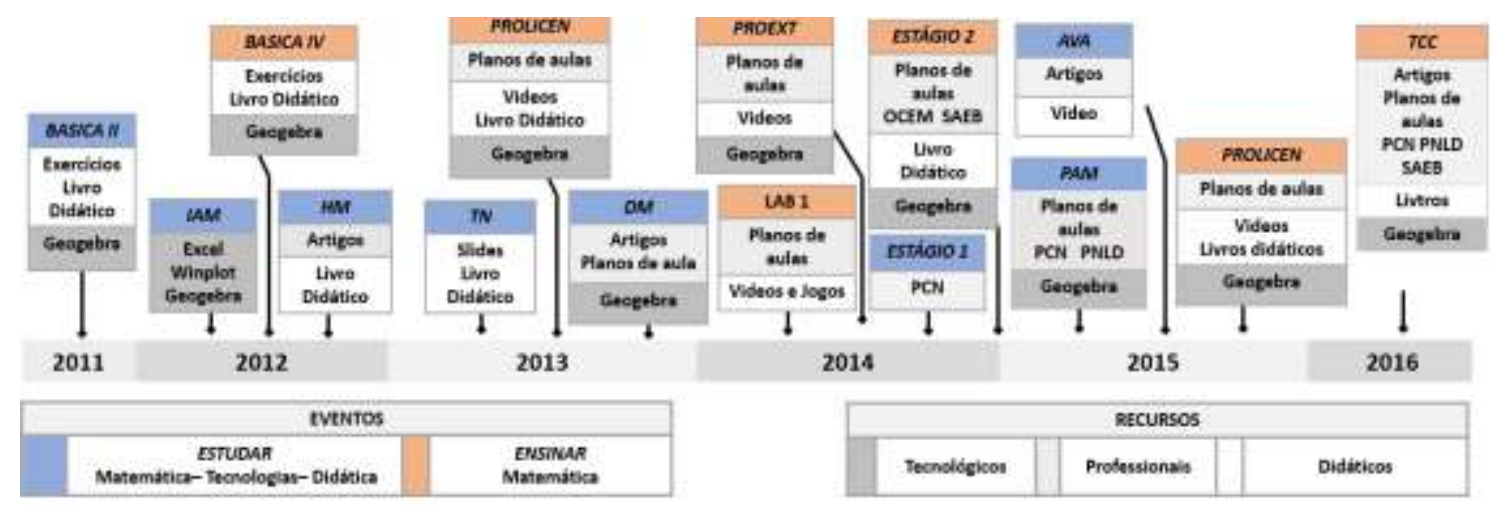

Figura 3 - Representação inferida para a trajetória documental de Cecília.

Resumidamente, representamos eventos (disciplinas curriculares e projetos institucionais) relacionados ao estudo (matemática, tecnologias e didática) em azul, e ao 
Cibelle Assis, Verônica Gitirana, Luc Trouche

A metamorfose do sistema de recursos de licenciandos em matemática na formação inicial

ensino (matemática) em laranja, além dos recursos utilizados nestas situações (tecnológico, profissional e didático), em tons de cinza.

Observamos como o Geogebra esteve presente na sua formação participando de diferentes eventos, sendo utilizado como recurso para estudar e ensinar. A licencianda também destacou na sua trajetória a importância das disciplinas de estágio supervisionado (Estágio 1 e 2) e de projetos institucionais (Prolicen e Proext).

Analisando os Grupos de Recursos enviados por Cecília, compomos um SREst e um SREns. Esses recursos foram identificados nas proposições de tarefas usadas em situações de estudo (eventos Básica II e Básica IV, por exemplo) ou produzidas pela licencianda em situações de ensino (eventos Prolicen e TCC, por exemplo) entre os anos de 2011 e 2016. Nesta perspectiva apresentamos elementos da dimensão conteúdo da metamorfose de Cecília:

- SREstudar: Livros, livros didáticos, lista de exercícios, materiais manipulativos, Geogebra e outras TIC.

- SREnsinar: Livros, livros didáticos, planos de aula, materiais manipulativos, Geogebra, vídeos para aulas, datashow, artigos sobre Geometria Dinâmica, Referenciais curriculares (PCN) e documentos nacionais como SAEB e Guia do PNLD.

Sobre a dimensão estrutura, inferimos papéis para tais recursos e, portanto, de seus usos. No caso da Geogebra e suas ferramentas assim como os livros didáticos e as listas de exercícios, seus papéis foram ampliados passando de recursos para estudar matemática ou realizar uma tarefa matemática para conceber, estruturar tarefas e apoiar a atividade de "seus" alunos em uma aula de matemática. Neste caso chamamos tais recursos de recursos de transição. Também identificamos novos papéis que vieram com a introdução de novos recursos, caso dos artigos científicos sobre Geometria Dinâmica, os referenciais curriculares (PCN) e alguns dos documentos nacionais como SAEB e Guia do PNLD. Chamamos tais recursos de recursos profissionais.

Por fim, sobre a dimensão natureza que enseja conhecimentos e recursos, consideramos uma situação profissional vivida por Cecília durante o evento TCC (elaboração do Trabalho de Conclusão de Curso): concepção e implementação de uma aula sobre classificação de triângulos quanto aos lados utilizando o Geogebra. A aluna discutiu possibilidades da geometria dinâmica para o estudo da classificação de triângulos) em uma turma do $8^{\circ}$ Ano do Ensino Fundamental de uma escola pública do 
Cibelle Assis, Verônica Gitirana, Luc Trouche

A metamorfose do sistema de recursos de licenciandos em matemática na formação inicial

município de Guarabira no Estado da Paraíba. A partir da implementação da aula registrada por vídeo, dos documentos plano da aula e roteiro de atividades, inferimos um esquema de ensino e alguns invariantes operatórios:

- Esquema de ensino: Classificar triângulos (equilátero, isósceles, escaleno) usando o Geogebra.

- Invariantes operatórios: Cada família de triângulos (equilátero, isósceles e escaleno) possui um procedimento diferente de construção no Geogebra; o procedimento de construção no Geogebra usa propriedades matemáticas; construções robustas no Geogebra preservam as propriedades de cada família de triângulos (equilátero, isósceles e escaleno); cada construção robusta oferece um número ilimitado de triângulos.

O esquema revelou como a licencianda confia no Geogebra para obter e criar exemplos de triângulos, e para apresentar a classificação dos triângulos aos alunos da escola. Associados a este esquema, alguns invariantes operatórios podem ser chamados de resistentes e flexíveis. De fato, são resistentes porque estavam presentes em seu esquema de estudo (Ex. A identificação de propriedades dos triângulos pode ser realizada a partir da análise de vários exemplos como no livro) e flexíveis, devido ao desenvolvimento de esquemas de ensino através da integração entre recursos antigos e novos (Ex. ela sabe que cada construção robusta feita em Geogebra oferece um número ilimitado de triângulos mais do que aqueles presentes no livro didático).

\section{Conclusões: A metamorfose e a formação inicial}

Em síntese, este estudo de caso contribuiu para a formação de uma percepção diferenciada para os recursos e seus usos na formação inicial de professores de Matemática. Uma vez observada a presença e a influência destes no processo de metamorfose dos estudantes, interrogamos os recursos utilizados e produzidos por eles, pelos professores dos cursos de formação e até mesmo das escolas onde os licenciandos realizaram algumas de suas atividades. Nesta pesquisa a Trajetória Documental apresentou-se como um instrumento para a representação de um panorama geral da formação do estudante e do próprio curso.

Tais observações fazem emergir outros questionamentos no contexto da formação inicial: "como motivar o desenvolvimento de novos esquemas de utilização e 
Cibelle Assis, Verônica Gitirana, Luc Trouche

A metamorfose do sistema de recursos de licenciandos em matemática na formação inicial

de invariantes operatórios associados aos recursos?", "que repertório de recursos e de usos estão sendo apresentados aos licenciandos através das disciplinas e projetos constituintes da formação?". Tais questões nos fazem refletir também sobre a necessidade do acompanhamento da metamorfose dos licenciandos e suas especificidades pela equipe de professores envolvidos na formação.

\section{Referências}

ADLER, J. Conceptualising resources as a theme for teacher education. Journal of Mathematics Teacher Education, n. 3, p. 205-224, 2000.

BELLEMAN, F.; TROUCHE, L. Compreender o trabalho do professor com os recursos de seu ensino, um questionamento didático e informático. I Simpósio Latino-Americano de Didática da Matemática, Mato Grosso do Sul. Anais. 2016. Disponível em:< https://drive.google.com/file/d/0B6OphkgfrkD3eGRlSW1iVHg3YjQ/view>.

BESNIER, S. Le travail documentaire des professeurs à l'épreuve des ressources technologiques. Le cas de l'enseignement du nombre à l'école maternelle (Doctoral dissertation). Disponível em: <https://filesender.ens-lyon.fr/?vid=711f5501-c53e-7089139d-00004448e9a4>.

GOIGOUX, R.: VERGNAUD, G. Schémes professionnels. AIRDF, n. 36, v. 1, p. 7-10. 2005.

GUEUDET, G.; TROUCHE, L. Du travail documentaire des enseignants: genèses, collectifs, communautés. Le cas des mathématiques. Education et didactique, Rennes, n. 2, v. 3, p. 7-33. 2008.

GUEUDET, G.; TROUCHE, L. Towards new documentation systems for mathematics teachers? Educational Studies in Mathematics, n. 71, v. 3, p. 199-218. 2009.

GUEUDET, G.; TROUCHE, L. Teachers' work with Resources: Documentational Geneses and Professional Geneses. In: G. GUEUDET; B. PEPIN; L. TROUCHE. (Eds.). From text to "lived" resources mathematics curriculum materials and teacher development. Dordrecht: Springer, 2012. p. 23-41.

PONTE, J.; BRUNHEIRA, L. Analysing practice in preservice mathematics teacher education. Journal of Mathematics Teacher Development, n. 3, p. 16-27, 2001.

ROCHA, K.;TROUCHE, L. Documentational trajectory: a tool for analyzing the genesis of a teacher's resource system across her collective work. In: T. DOOLEY; G. GUEUDET (Eds.). (2017, in preparation). Proceedings of the tenth congress of the european society for research in mathematics education (CERME10, February $1-5$, 2017). Dublin, Ireland: DCU Institute of Education and ERME.

VERGNAUD, G. Teoria dos campos conceituais. Seminário Internacional de Educação Matemática do Rio de Janeiro, 1., 1993, Rio de Janeiro. Anais. Rio de Janeiro: UFRJ Projeto Fundão, Instituto de Matemática, p. 1-26. 
Cibelle Assis, Verônica Gitirana, Luc Trouche

A metamorfose do sistema de recursos de licenciandos em matemática na formação inicial

YIN, R. Estudo de caso: planejamento e métodos. Porto Alegre: Bookman, 2005. 